\title{
The revival of bare-knuckle boxing: is this early form of pugilism actually more dangerous than modern boxing?
}

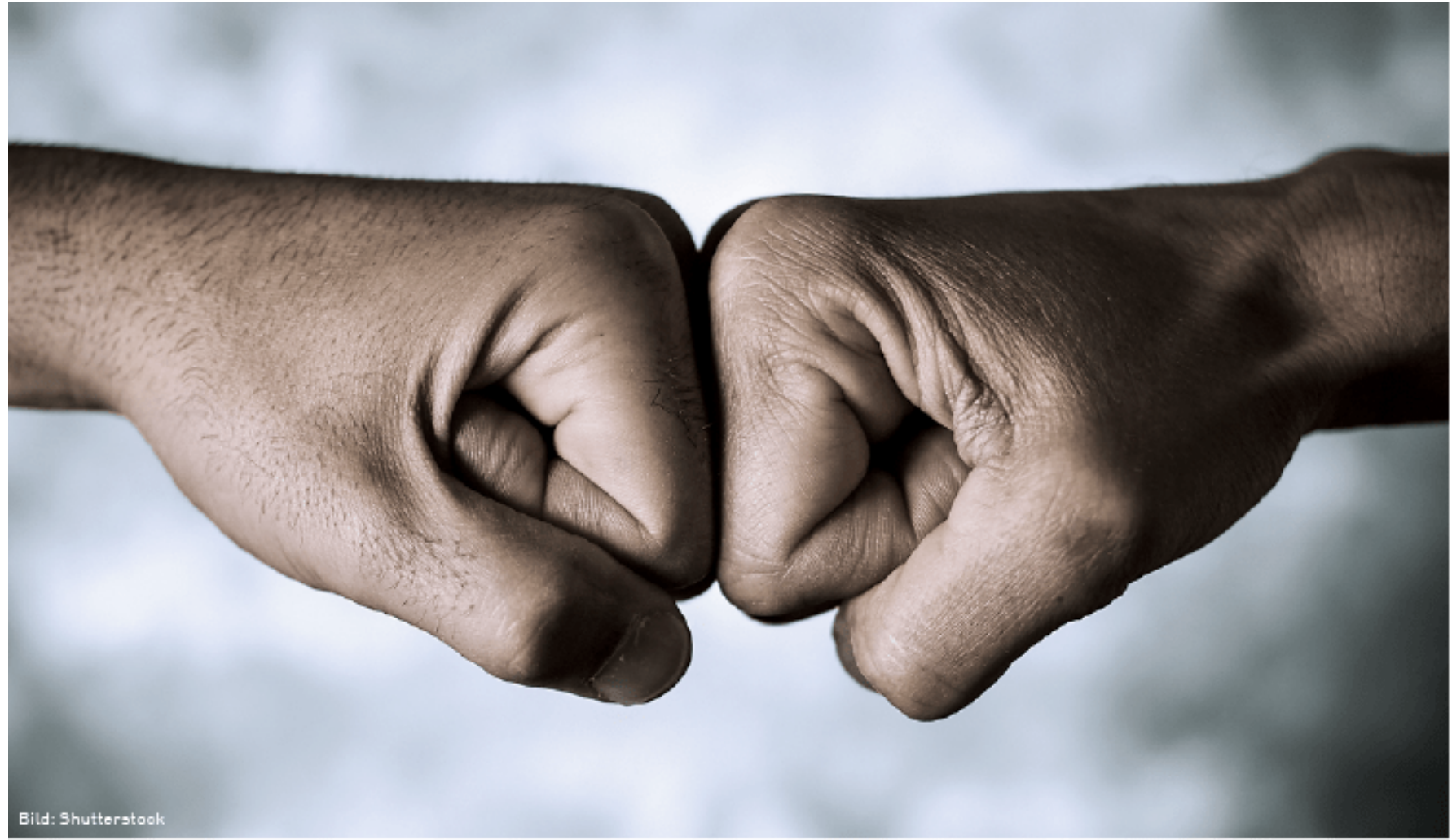

Stanbouly Dani, BS1; Chuang Sung-Kiang, DMD, MD, DMSc2

1 Dental Student, Columbia University College of Dental Medicine, New York, NY

2 口Clinical Professor, Department of Oral and Maxillofacial Surgery, University of Pennsylvania, School of Dental Medicine, Philadelphia, PA; Private Practice, Brockton Oral and Maxillofacial Surgery Inc.; Attending, Department of Oral and Maxillofacial Surgery, Good Samaritan Medical Center, Brockton, MA; Visiting Professor, Department of Oral and Maxillofacial Surgery, Kaohsiung Medical University, School of Dentistry, Kaohsiung, Taiwan

Boxing is a sport with a clear objective: to hit and not get hit. Despite all the rules and regulations that have been conceived for safety purposes over time, boxing will always be a hurting business at the end of the day. The sweet science is unique from all other contact sports, like hockey and football, in that the damage 
inflicted on your opponent is deliberate [1].

The sport of boxing is an ancient one, dating back to the Greek and Roman empires. The earliest accounts of boxing describe a different scene from today, that which is marked by the absence of gloves as boxers fought with their bare fists. In 1867, John Sholto Douglas, the ninth Marquis of Queensberry, effectively put an end to the era of bare-knuckle boxing by introducing a set of rules, the most important of which mandated the use of leather gloves. It was believed that gloves would distribute the blow and spread its impact. Hence, the recipient of a punch is less likely to be blinded, lose their teeth, or, most critically, break the bones of their maxillofacial skeleton than if the force was finely concentrated within the serrated surface area of the aggressor's knuckles [1]. Many studies attest to of the efficacy of the Queensbury rule. A study on all professional boxing bouts within a 16-year timeframe in an organized federation recorded a total of 107 injuries were recorded in 427 fight participations. The most common type of injury was wound/laceration (66.4\%). Fracture of the maxillofacial skeleton, in contrast, was one of the least common injuries, comprising merely five of the injuries (4.7\%) [2].

But could boxing gloves have also made the sport more dangerous in other respects? While the introduction of boxing gloves makes it harder to precipitate different forms of maxillofacial trauma, it inadvertently increased the likelihood of injuring a less conspicuous organ, the brain, as a tradeoff for the more obvious organ, the face. While boxing gloves certainly cushion the fists and may protect a fighter from many acute injuries, such as deep lacerations or orbital bone fractures, it doesn't protect a fighter from chronic brain injuries that only accumulate over the span of a fighter's career. In fact, gloves make matters worse by adding more mass of the fist, increasing the net momentum delivered to the opponent's head with each punch and ultimately rattling the delicate brain inside the skull [1]. Additionally, the skull bones are physically stronger than the metacarpals. Thus, the bare-knuckle boxer risks damaging his fists more than his opponent's skull with a forceful punch. The hand-protection offered by gloves therefore encourages a modern (i.e., gloved) boxer to punch with a significantly higher degree of power than otherwise [1].

Chronic traumatic encephalopathy (CTE), also known as pugilistic dementia, is a neurological condition that occurs in professional boxing as a result of repetitive subconcussive blows to the head over the course of a career [1]. It is characterized by a constellation of symptoms: dysarthria (i.e., slurred speech) and gait ataxia (i.e. disequilibrium) are present in almost all cases of the condition. The condition ranges in severity depending on the amount of damage accrued by the brain, which is more or less congruent to the length of the boxer's career and the number of bouts they had participated in [3].

Recently, there has been a legal reemergence of bare- knuckle boxing in effort by the promotion: Bare Knuckle Fighting Championship (BKFC). This form of boxing is governed by several rules distinct from modern boxing. Fighters are not allowed to wrap their hands to within one inch of the knuckle in order to fully expose it for offense. Fights are only two minutes per round, compared to three in modern boxing, and each bout is only 5 rounds in length, compared to 12 in modern boxing. If a fighter is cut and the bleeding cannot be controlled, the referee will stop the fight [4]. With lighter fists and shorter fights, bareknuckle boxing is theoretically less dangerous to the brain than modern boxing. Nevertheless, does this benefit compensate the increased risk of maxillofacial trauma?

Many measures can be taken to protect fighters in both forms of boxing. Lighter gloves transmit less momentum to the head and their use should be encouraged in modern boxing [5]. Body shots should be worth as many points as head shots in the scoring system for both forms of boxing in an effort to 
encourage boxers to deliver just as much body shots as they do head shots. The fact that head shots are favored by judges explains the predominance of head shots over body shots in a typical boxing bout [1]. While several studies have demonstrated the presence of CTE in boxers, it is, at least, reassuring to know that a lot has changed since these studies were conducted. Professional boxing exposure has significantly decreased since the 1930s, both in terms of career length (mean of 19 years reduced to 5 years) and bout number (mean of 336 fights reduced to 13 fights). The length of bouts has also decreased through time. While boxing matches could have stretched up to 20 rounds in the past, the maximum number of permitted for any professional fight today is 12 [1]. Finally, it was common practice for retired boxers to become sparring partners or even tent boxers at carnivals in the 1930s-1950s, further increasing the net brain damage accrued over their lifetime. Hence, we can assume that the incidence of CTE amongst boxers in that era must have been significantly higher than that of boxers today [3]. This reassuring assumption, however, should not negate promoting the aforementioned interventions for the safety of the fighters. Each form of boxing is undoubtedly dangerous in its own way. Contemporary (i.e., gloved) boxing is especially injurious to the brain: the gloves increase the momentum per punch, encourage harder punches by virtue of protecting the boxer's hands, and prolong fights due to the relative infrequency of maxillofacial trauma (i.e., lacerations, skull fractures). Bare-knuckle boxing is particularly damaging to the face: the absence of any form of padding between a boxer's knuckles and the opponents face renders maxillofacial fractures and dental avulsions more likely. While the health implications of modern boxing is well established in the literature, the same is not true for bare-knuckle boxing since the sport's legal revival is very recent. Hence, there is a lot of potential research to quantify the severity of maxillofacial trauma from bare-knuckle boxing.

\section{Conflicts of Interest}

There are no conflicts of interest declared by any of the authors. This study received no commercial funding.

\section{Funding}

No funding obtained.

\section{Corresponding author}

Dani Stanbouly, BS

College of Dental Medicine Columbia University

630 West 168th Street

New York, NY 10032

T: 616-375-5924

F: 212-305-5958

ds3840@cumc.columbia.edu [

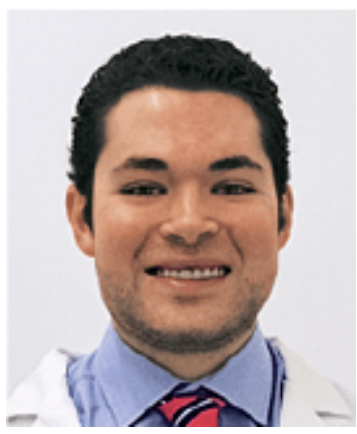




\section{References}

1. THE HISTORY OF BAREKNUCKLE BOXING

https://historyofbkb.weebly.com/the-history-of-bkb.html (accessed February 20, 2021)

2. T R Zazryn, C F Finch, P McCrory. A 16 year study of injuries to professional boxers in the state of Victoria, Australia. Br J Sports Med. 2003;37:321-324

3. McCrory, Paul, et al. The evidence for chronic traumatic encephalopathy in boxing. Sports Medicine. 2007;37(6):467+

4. ABOUT BARE KNUCKLE FC.

https://www.bareknuckle.tv/about (accessed February 24, 2021)

5. Rudd, S., Hodge, J., Finley, R., Lewis, P., \& Wang, M. Should we ban boxing? BMJ : British Medical Journal. 2016;352 